\title{
Anticancer potential of rosmarinic acid and its improved production through biotechnological interventions and functional genomics
}

\begin{abstract}
Rosmarinic acid (RA) is a highly valued natural phenolic compound that is very commonly found in plants of the families Lamiaceae and Boraginaceae, including Coleus blumei, Heliotropium foertherianum, Rosmarinus officinalis, Perilla frutescens, and Salvia officinalis. RA is also found in other members of higher plant families and in some fern and horned liverwort species. The biosynthesis of RA is catalyzed by the enzymes phenylalanine ammonia lyase and cytochrome P450-dependent hydroxylase using the amino acids tyrosine and phenylalanine. Chemically, RA can be produced via methods involving the esterification of 3,4-dihydroxyphenyllactic acid and caffeic acid. Some of the derivatives of RA include melitric acid, salvianolic acid, lithospermic acid, and yunnaneic acid. In plants, RA is known to have growth-promoting and defensive roles. Studies have elucidated the varied pharmacological potential of RA and its derived molecules, including anticancer, antiangiogenic, antiinflammatory, antioxidant, and antimicrobial activities. The demand for RA is therefore, very high in the pharmaceutical industry, but this demand cannot be met by plants alone because RA content in plant organs is very low. Further, many plants that synthesize RA are under threat and near extinction owing to biodiversity loss caused by unscientific harvesting, overcollection, environmental changes, and other inherent features. Moreover, the chemical synthesis of RA is complicated and expensive. Alternative approaches using biotechnological methodologies could overcome these problems. This review provides the state of the art information on the chemistry, sources, and biosynthetic pathways of RA, as well as its anticancer properties against different cancer types. Biotechnological methods are also discussed for producing RA using plant cell, tissue, and organ cultures and hairy-root cultures using flasks and bioreactors. The recent developments and applications of the functional genomics approach and heterologous production of RA in microbes are also highlighted. This chapter will be of benefit to readers aiming to design studies on RA and its applicability as an anticancer agent.
\end{abstract}

Keyword: Anticancer activity; Bioreactors; Biotechnology; Plant tissue culture; Rosmarinic acid 